\title{
Utopía y adolescencia
}

\section{María José Gómez Cantos}

IES Marxadella, Torrent-Valencia, España, gomezcantos@hotmail.com

\begin{abstract}
Resumen
La asignatura de filosofía en el Bachillerato a menudo es considerada por los estudiantes como excesivamente teórica y con escasa utilidad. Con el objetivo de incorporar una perspectiva de estudio más conectada con la vida cotidiana y estimular una manera de pensar el mundo más amplia y crítica, se propone la elaboración de materiales didácticos que fomenten el pensamiento creativo, el aprendizaje significativo de los contenidos del currículum y el acercamiento a la investigación social cualitativa.

En el presente trabajo se plantea a los alumnos la investigación, a partir de la técnica de análisis de contenido, de las utopías sociales que ellos mismos han elaborado. De esta manera, después de estudiar los contenidos básicos de la asignatura de filosofía, se pide a los estudiantes que diseñen una sociedad utópica (un mundo justo y feliz). A continuación, el material elaborado se analiza con la técnica del análisis de contenido. Por último, se establecen, exponen y discuten las conclusiones.
\end{abstract}

Palabras clave: Utopía, antiutopía, adolescencia, filosofía

\section{Introducción}

El presente artículo tiene como objetivo exponer detalladamente el diseño de una unidad didáctica sobre el pensamiento utópico para la asignatura de Filosofía del Bachillerato. Los contenidos de la unidad se centran en las utopías sociales y diferentes aspectos de las mismas. Para desarrollar estos contenidos planteamos una serie de actividades que buscan que los alumnos aprendan filosofía pero que también aprendan a filosofar y que sean atractivas y motivadoras. Para ello partimos del cine y la literatura juvenil que está de moda entre los jóvenes y que recrea antiutopías o utopías negativas. Al mismo tiempo, los estudiantes inventarán utopías sociales, que posteriormente analizarán para establecer cuáles son las injusticias del orden social actual y qué alternativas pueden proponerse. 
Dicha unidad didáctica busca favorecer la consecución de los objetivos generales del Bachillerato. Tal y como señala el Decreto del Consell 87/2015, de 5 de junio en el que se establece el currículum del Bachillerato, éstos son proporcionar al alumnado formación, madurez intelectual y humana, conocimientos y habilidades que les permitan desarrollar funciones sociales e incorporarse a la vida activa con responsabilidad y competencia.

El aprendizaje basado en competencias ha de caracterizarse por su transversalidad, su dinamismo y su carácter integral. Para favorecer estos procesos hemos elaborado una unidad didáctica que se trabajará como último tema de la asignatura de Filosofía de $1^{\mathrm{o}}$ Bachillerato y entrelazará los contenidos de la materia de Filosofía y una técnica de investigación social, el análisis de contenido. La interdisciplinariedad implica la interacción de varias disciplinas, entendida como el diálogo y la colaboración de éstas para lograr la meta de un nuevo conocimiento (Van del Linde, 2007). Nuestra estrategia didáctica se basa, además, en el aprendizaje significativo, la creatividad y la resolución de problemas.

Los materiales didácticos presentados los consideramos adecuados a la etapa del desarrollo psicológico de los alumnos y a su contexto social. Por una parte, el adolescente empieza a adquirir la capacidad de explorar consciente y creativamente los significados más profundos de la realidad pero necesita experiencias para ejercitar dicha capacidad. Por otra parte, vivimos en una sociedad individualizada que se refleja en la literatura y el cine que engancha a los adolescentes: utopías negativas que muestran sociedades totalitarias y muy tecnologizadas donde los jóvenes luchan entre ellos para sobrevivir. En este sentido, la invención y el análisis de utopías sociales persigue la reflexión sobre los problemas y la propuesta de soluciones, desde la perspectiva del grupo social y no del individuo particular aislado de todo vinculo social.

Planteamos la aproximación didáctica desde filosofía y las técnicas de investigación social, contenidos todos ellos que forman parte del currículum de Filosofía en $1^{\circ}$ Bachillerato. Estos contenidos han sido estructurados en tres apartados que se secuencian en cinco semanas lectivas con un total de 15 sesiones.

\section{Propuesta didáctica}

La unidad se estructura en tres apartados. En primer lugar, se plantea el trabajo que tienen que desarrollar los alumnos individualmente, fuera del aula, durante dos semanas: la invención de una utopía siguiendo un guión, proporcionado por la profesora, basado en el patrón universal de Marvin Harris (1987). Buscamos con ello favorecer la asimilación de los contenidos estudiados en las clases de filosofía a lo largo del curso y su aplicación en las plasmación de un proyecto global de sociedad en base a los valores de justicia y 
felicidad. Este trabajo se realizará en casa de manera individual. Se pretende fomentar la creatividad y la reflexión individual y en soledad.

Un segundo bloque, en el que se desarrollan y trabajan los aspectos teóricos del tema a partir de una serie de actividades. Este trabajo se lleva a cabo en el aula, al mismo tiempo que los alumnos inventan en sus casas la utopía social.

Finalmente, se pasa al estudio de las utopías elaboradas, mediante la técnica del análisis de contenido. Esta tarea se realiza en el aula y por grupos. Seguidamente, se exponen y discuten las conclusiones de cada grupo. El último bloque se llevará a cabo a lo largo de tres semanas.

\subsection{Introducción y elaboración de un mundo utópico}

Se explica a los alumnos que se va a trabajar el tema de la utopía, para lo cual, van a tener que asumir dos roles, primero el de filosofo y después el de científico social. En un primer momento, como filósofos van a utilizar libremente todo lo estudiado durante el curso para inventar una utopía. A continuación, desempeñarán el papel de científicos sociales y analizarán dichas utopías con la técnica del análisis de contenido. El objetivo es averiguar cuáles son los rasgos de las utopías de los adolescentes de principios del siglo XXI.

De esta manera, los estudiantes, lo primero que tienen que hacer es construir una utopía social, esto es, un mundo feliz y justo. Para ello, seguirán los siguientes puntos basados en el patrón universal de Marvin Harris (1980:134-135):

1. Localización: ecosistema

2. Las prácticas empleadas para incrementar o limitar la producción básica de subsistencia, en especial la producción de alimentos y otras formas de energía, dadas las restricciones y oportunidades provocadas por una tecnología específica que interactúa con un hábitat determinado. Tecnología de subsistencia. Relaciones tecno-ambientales. Pautas de trabajo.

3. Estructura familiar. Tipos de familia. División doméstica del trabajo. Socialización doméstica, educación. Edad y roles sexuales. Jerarquías de disciplina doméstica, sanciones. Fertilidad, natalidad y mortalidad. Nutrición infantil. Anticoncepción, aborto, infanticidio.

4. ¿Quién gobierna? ¿Cómo? ¿Por qué? La organización entre bandas, poblados, jefaturas, estados e imperios. Organización política, Sistema de impuestos, tributos. Socialización política. Grupos sociales: clase, casta, jerarquías urbanas, rurales. Disciplina, control policial y militar. Guerra. 
5. Sistema jurídico: Los derechos y deberes fundamentales que tendrían las personas que vivan en vuestro mundo. Habrá cosas permitidas y cosas prohibidas. Estos permisos y prohibiciones también nos hablan de cómo está diseñada la sociedad. Sistema de sanciones

6. ¿Qué se aprende? ¿Cómo? ¿Por qué? Sistema educativo. Nivel de desarrollo científico

7. Otros aspectos: Arte, música, danza, literatura, religión y rituales propaganda, deportes, juegos, hobbies.

\subsection{Contenidos teóricos: la utopía}

En este apartado se trabaja, a partir de una serie de actividades, el concepto de utopía: su definición y rasgos básicos, la relación con el contexto histórico, su evolución y la situación actual de agotamiento.

\subsubsection{Actividad 1: La utopía y sus rasgos definitorios}

Los alumnos leen un texto que trata sobre la utopía en Tomas Moro (Cortina, 1996:346). A partir del texto, extraen el contexto histórico del surgimiento de la utopía de Tomas Moro, las diferencias con otros autores de filosofía política, la critica a la sociedad en la que vive, el origen de todos los problemas de su sociedad, según Moro y los rasgos básicos de la sociedad utópica que propone.

Seguidamente, se explican los rasgos básicos de las utopías y se comentan, haciendo especial énfasis en el tema de la doble función de las utopías: la de crítica social y la función constructiva.

\subsubsection{Actividad 2: La utopía a lo largo de la historia}

Se visiona en clase la película Divergente (2014), película estadounidense basada en la novela del mismo nombre escrita por Veronica Roth y dirigida por Neil Burger. Un film que han visto la mayor parte de los alumnos y que les agrada. Divergente trata de una sociedad futura, donde los habitantes están divididos en grupos según sus personalidades. La protagonista descubre que es una divergente y tiene rasgos de todos los grupos. Además, averigua un plan de los gobernantes para destruir, a quienes con como ella, son divergentes, dado que amenazan el orden social establecido.

Tras el visionado de la película, se trabaja con el método de la asamblea (Caruana, 2007:143-148) con todo el grupo-clase, y se compara el modelo de sociedad que propone Platón en la República, con la organización política y social de Divergente. Interesa que los estudiantes infieran las semejanzas, pero sobre todo las diferencias, entre un modelo de sociedad de un autor de la Grecia Clásica y un modelo de sociedad de una autora de la 
modernidad muy avanzada. Importa resaltar la cuestión de que en Platón el ser humano se considera social por naturaleza, se constituye por y para la vida en sociedad. Platón intenta establecer el puente entre la perspectiva individual del ser humano y la social. Además, la finalidad del Estado es la felicidad y el bien de los ciudadanos. Por el contrario, la organización social que aparece en Divergente refuerza la idea de que la búsqueda de la identidad propia, supone tener que luchar contra la sociedad, la cual está regida por un gobierno totalitario que sólo busca aumentar su poder. En Divergente se dibuja una organización social totalmente destructiva para el ser humano, que lo limita y le causa sufrimiento.

\subsubsection{Actividad 3. Las antiutopías en el mundo actual.}

Para finalizar este bloque se plantea la siguiente pregunta a los alumnos: ¿Por qué están de moda las antiutopías entre los adolescentes actuales? Las respuestas a esta cuestión se elaborarán siguiendo la técnica "el arte de preguntarse mutuamente" que propone Brenifier (2005:75-84). Con esta dinámica buscamos que los alumnos construyan sus propios razonamientos a partir de todo lo trabajado en clase. Aquellos alumnos que no participan oralmente son los encargados de realizar la síntesis de todas las tesis y argumentos planteados por sus compañeros. Finalmente, se leen dichas síntesis y se reflexiona sobre algunas hipótesis, como por ejemplo, que el surgimiento de las antiutopías está relacionado con el contraste entre el gran desarrollo científico y tecnológico que experimentan las sociedades humanas y el escaso conocimiento y control de los procesos sociales (Elias, 1998); o que el éxito de las utopías negativas está influido por la idea de que los intentos de aplicar las ideas utópicas han acabado en violencia y totalitarismos y es imposible imaginar una alternativa al sistema económico capitalista.

\subsection{Aplicación de la técnica análisis de contenido al material elaborado por los alumnos}

Este bloque se introduce haciendo referencia a los contenidos estudiados durante el curso sobre el método científico, las diferencias entre ciencias naturales y sociales y los diferentes metodologías de investigación (cuantitativas y cualitativas).

Seguidamente, se explica la técnica de análisis de contenido siguiendo a López Aranguren (1994: 461-491) y se aplica al fenómeno que vamos a investigar: los rasgos básicos de los mundos utópicos de los adolescentes. Este trabajo se lleva a cabo en dos etapas: en la primera, se trabaja con todo el grupo clase y en la segunda, se realiza trabajo en grupos.

Todo el grupo clase realiza conjuntamente las primeras fases del análisis de contenido y se anota en la pizarra. Concluyendo los siguientes puntos: 
El fenómeno de interés: ¿Cuáles son los rasgos básicos de los mundos utópicos de los adolescentes?

Los datos: utopías elaboradas por estudiantes de $1^{\circ}$ Bachillerato con edades comprendidas entre los 16 y 18 años, de clase social media y media-baja, de una población de 80600 habitantes, del área metropolitana de Valencia.

El contexto de los datos: crisis económica, violencia machista, corrupción política, problemas ecológicos, desarrollo tecnológico, precariedad laboral, universalización del uso de las TICS...

El objetivo del análisis: En este caso sería la descripción. Se trataría de una investigación de carácter exploratorio en la se pretende desentrañar las injusticias del orden social según los estudiantes de $1^{\circ}$ Bachillerato y ver qué alternativas ofrecen para la organización de la sociedad y el modo que pueden llevarse a cabo.

Unidades de análisis: distinguimos entre unidades de muestreo (cada uno de los mundos utópicos creados por los alumnos); y las unidades de registro (cada uno de los ítems desarrollados en el creación de la utopía). En concreto trabajamos con 7 unidades de registro: localización, modo de subsistencia, estructura familiar, sistema político, sistema jurídico, sistema educativo y lo que hemos englobado en "otros aspectos".

La siguiente fase del análisis de contenido se realiza por grupos. Se divide la clase en 7 grupos (tantos como unidades de registro) y cada grupo analizará una unidad de registro, formando las categorías. Interesa el contenido en cuanto sustancia o fondo. Se trata de comparar los contenidos de las comunicaciones de cada uno de los alumnos. Buscamos semejanzas y diferencias respecto a lo que se consideran las injusticias del orden social en el que vivimos, las alternativas a dichas injusticias y la manera de implementar las alternativas en la sociedad. Al mismo tiempo, se realizan inferencias sobre los valores y creencias que subyacen a las ideas expresadas en las unidades de registro.

Cada grupo tiene que elaborar un cuadro donde resuma sus conclusiones que se expondrán y comentarán en clase.

\subsection{Evaluación}

La última actividad tiene como finalidad evaluar el proceso de enseñanza-aprendizaje y comprobar si se han alcanzado los objetivos propuestos. Entre ellos:

- La asimilación de los contenidos estudiados a lo largo del curso

- Crítica al contexto social desde la perspectiva del grupo 
- La aportación de soluciones colectivas a problemas sociales

- La aplicación de una técnica de investigación social

Para ello se propone a los alumnos la elaboración de un texto individual con el título "Las utopías de los adolescentes de principios del siglo XXI". En dicho texto se sintetiza la información obtenida a partir de todo el trabajo previo. El texto tiene recoger los siguientes temas: contexto histórico de los estudiantes de $1^{\circ}$ Bachillerato, referencia a las semejanzas y diferencias con las creaciones de otros autores de su época (los creadores de las antiutopías), la critica a la sociedad actual, la causa o causas de los problemas sociales detectados y los rasgos básicos de un mundo feliz y justo.

\section{Conclusiones}

Los materiales didácticos que hemos presentado pueden aproximarnos a diversas metas de la práctica docente: estudiar los grandes filosofos, relacionarlos con problemas actuales y fomentar el pensamiento creativo y crítico. Al mismo tiempo, el alumnado realiza tanto trabajo individual como en grupo, desarrolla las competencias de escritura y también el dialogo en clase. Además, se fomentan las habilidades del pensamiento como analizar, comparar y argumentar. Por otra parte, cuando esta unidad didáctica se ha implementado durante varios cursos, resulta muy interesante observar como los cambios sociales - por ejemplo, la ley del matrimonio homosexual o la profunda crisis económica- se proyectan en las utopías de los adolescentes. No obstante, una unidad didáctica de este tipo puede plantear un problema de tiempo, necesita para su desarrollo varias semanas lectivas y el currículo de Filosofía en el Bachillerato es muy extenso. En este caso, se podría prescindir del análisis de contenido y que los alumnos expongan y comenten en clase sus mundos felices y justos.

Desde la perspectiva de los estudiantes puede ser especialmente motivador tener que inventar una utopía. Los adolescentes son especialmente críticos con la sociedad y están desarrollando su identidad personal por ello, les puede resultar atractivo diseñar un mundo completamente a su gusto. Además, al compararlo con el de sus compañeros, se dan cuenta de que comparten muchas ideas, que sus mundos ideales no son tan distintos y que, seguramente, para vivir en nuestros mundos ideales nosotros como personas también tendríamos que cambiar. 


\section{Referencias}

Borobia, R. (2004) La hipótesis en estudios cualitativos. El caso de la inducción analítica en una investigación sobre adolescencia, en Revista Pilquea $\mathrm{n}^{0}$ 6. Recuperado de http://www.scielo.org.ar/scielo.php?script=sci arttext\&pid=S1851-31232004000100005

Brenifier, O. (2005). El diálogo en clase, Santa Cruz de Tenerife, Ediciones Idea.

Caruana, A. (Coord.) (2007). Programa de educación emocional para la prevención de la violencia. Valencia, Ed. Generalitat Valenciana.

Cortina, A. (1994). Filosofía, Madrid, Alianza.

Decreto 87/2015, de 5 de junio, del Consell, por el que se establece el currículo y desarrolla la ordenación general de la Educación Secundaria Obligatoria y del Bachillerato en la Comunidad Valenciana.

Elias, N. (1990). La sociedad de los individuos, Barcelona, Península.

Elias, N. (1998)."¿Cómo pueden las utopías científicas y literarias influir sobre el futuro?", en Weiler, Vera (ed.), Figuraciones en proceso, Santa Fé de Bogotá, Fundación Social.

Harris, M. (1987). Introducción a la antropología general, Madrid, Ed. Alianza.

Huxley, A. (2014). Un mundo feliz, Barcelona, Debolsillo.

Jameson, F. (2009). Arqueologías del futuro. El deseo llamado utopía y otras aproximaciones de ciencia ficción, Madrid, Akal.

Krippendorff, K. (1990). Metodología de análisis de contenido. Teoría y práctica, Barcelona, Paidós.

Linde, V. (2007). ¿Por qué es importante la interdisciplinariedad en la educación superior? Cuadernos de Pedagogía Universitaria Año 4. No. 8. 11 - 13. Pontificia Universidad Católica Madre y Maestra, Rep. Dominicana. Recuperado de http://slideflix.net/doc/189582/la-interdiscplinariedad$\% \mathrm{C} 2 \% \mathrm{BFalguna-vez-tehas-preguntado-por}$

López-Aranguren, E. (1994). "El análisis de contenido" en García Ferrando, M., Ibáñez, J, y Alvira, F. El análisis de la realidad social (pp. 461-491), Madrid, Alianza.

Moro, T. (2011). Utopía, Madrid, Ed. Espasa-Calpe.

Saavedra, M. (2004). Cómo entender a los jóvenes para educarlos mejor, México, Editorial Pax México.

Siegel, D. (2010). La mente en desarrollo. Cómo interactúan las relaciones y el cerebro para modelar nuestro ser, Bilbao, Desclée de Brouwer.

Siegel, D. (2011). Mindsight. La nueva ciencia de la transformación personal, Madrid, Paidós.

Siegel, D. (2014): Tormenta cerebral. El poder y el propósito del cerebro adolescente, Barcelona, Alba. 